\title{
Fabrication Process of Embedded Silicon Microchannel and Nozzle Structure for Liquid-delivering Atomic Force Microscopy Probe
}

\author{
Jinseo Hong, ${ }^{1}$ Yusuke Masuda, ${ }^{1}$ Yoshitaka Miura, ${ }^{1}$ \\ Takayuki Shibata, $^{2}$ and Takashi Mineta ${ }^{1 *}$ \\ ${ }^{1}$ Graduate School of Science and Engineering, Yamagata University, \\ 4-3-16 Jonan, Yonezawa, Yamagata 992-8510, Japan \\ ${ }^{2}$ Department of Mechanical Engineering, Toyohashi University of Technology, \\ 1-1 Hibarigaoka, Tempaku-cho, Toyohashi, Aichi 441-8580, Japan
}

(Received June 4, 2018; accepted December 3, 2018)

Keywords: atomic force microscope, hollow cantilever, microchannel, nozzle

In this paper, we demonstrate the novel fabrication processes of a dual $\mathrm{Si}$ atomic force microscopy (AFM) probe with embedded microchannels and a nozzle hole for liquid delivery function. The embedded microchannels and nozzle hole were separately located on the silicon surface to avoid the appearance of unnecessary crystalline (111) planes around the nozzle during the wet etching process for the probe tip formation, yet they were connected underneath the surface. The micronozzle was successfully formed at an inclined (111) plane of the Si AFM tip. The nozzle and embedded microchannels were successfully fabricated with under-surface connection with a path of $1 \mu \mathrm{m}$ diameter. The narrow trench opening of each microchannel was completely sealed by the combination of $\mathrm{Si}$ thermal oxidation and subsequent $\mathrm{SiO}_{2}$ sputtering deposition. After the sealing process, a microchannel of $1.5 \mu \mathrm{m}$ diameter was obtained. Applied pressure was estimated as $8 \mathrm{~Pa}$ in a microchannel of $1.5 \mu \mathrm{m}$ diameter and $2 \mathrm{~mm}$ length to obtain a flow rate of $0.5 \mathrm{fL} / \mathrm{s}$. A narrow-gapped Si dual tip with a nozzle and embedded microchannels were fabricated. The tip radius was sharpened to several nanometers through the combination of thermal oxidation and low-temperature thermal oxidation with the fabrication processes.

\section{Introduction}

Atomic force microscopy (AFM) technology has been widely used for imaging surface morphology with a sharp probe tip. ${ }^{(1)}$ Recently, AFM probes with a hollow structure have been developed for handling fluid in small volumes. ${ }^{(2-6)}$ For dispensing femtoliter-order droplets to a sample surface, a $\mathrm{Si}$ chip with a $\mathrm{SiO}_{2}$ hollow cantilever and a pyramidal silicon nitride AFM probe tip has been developed. ${ }^{(2)}$ A hollow Si cantilever with an embedded microchannel and a sharp hollow $\mathrm{SiO}_{2}$ needle has also been developed to deliver molecules into cells. ${ }^{(3)}$ Also,

*Corresponding author: e-mail: mineta-t@yz.yamagata-u.ac.jp https://doi.org/10.18494/SAM.2019.2018 
a linear array of hollow cantilevers made of SiN film with a volcano-shaped tip, which had a pyramidal probe tip with a ring-shaped hole around the tip, has been developed for patterning arrays of molecules using ink diffusion from the probe tip to the surface. ${ }^{(4)}$

We aim for liquid sample delivery, such as enzyme delivery, at an aimed position of a target biomolecule, which is imaged with high resolution by a sharp AFM probe tip. However, contamination and damage on a probe tip during the delivery operation prevents subsequent high-resolution imaging. In order to realize both liquid sample delivery to a biomolecule and in situ high-resolution imaging, a probe with two cantilevers with two separate functions is useful. In our previous work, we developed a dual AFM probe with narrow-gapped twin Si tips on twin cantilevers, which have a magnetostrictive film actuator for cantilever switching. ${ }^{(5)}$ We also fabricated a prototype of a dual AFM cantilever with a large deflectable structure to obtain a large deflection for cantilever switching. ${ }^{(6)}$

We have studied a basic fabrication process of a hollow Si cantilever with a microchannel. ${ }^{(7)}$ In this work, in order to enable the delivery of a liquid sample to a desired area and in situ highresolution imaging, we propose a whole fabrication process of a Si-embedded microchannel and a micronozzle hole on a Si probe tip for a dual AFM probe.

\section{Device Fabrication}

Figure 1 shows the concept of the narrow-gapped dual AFM probe with liquid delivery function. It consists of imaging and hollow cantilevers. The imaging cantilever has a sharp tip for high-resolution imaging, while the hollow cantilever has embedded microchannels $(1.5 \mu \mathrm{m}$ in diameter) and a tip $(\sim 8 \mu \mathrm{m}$ high) with a nozzle hole $(\sim 1 \mu \mathrm{m}$ in opening diameter) for liquid sample delivery. Magnetostrictive thin-film actuators are added on both cantilevers to switch the cantilevers between imaging and delivery functions. For example, when imaging the sample surface to determine the position for liquid delivery, the delivering cantilever is lifted away from the sample surface by magnetostrictive elongation. In contrast, when delivering a liquid

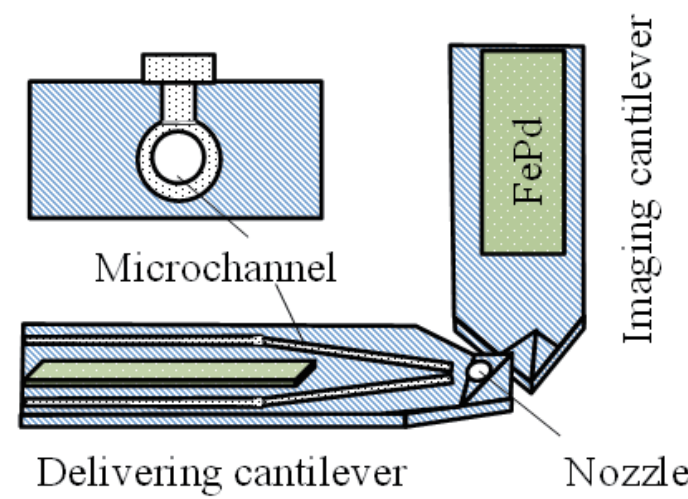

(a)

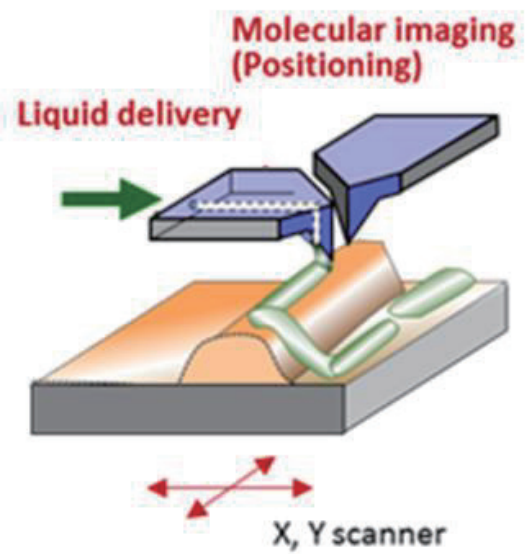

(b)

Fig. 1. (Color online) Concept of the (a) narrow-gapped dual AFM probe with (b) liquid delivery function. 
sample, the imaging cantilever is lifted away from the surface and the delivering cantilever approaches the sample surface and the liquid sample is deposited by externally applied pressure [Fig. 1(b)].

In this work, we focused on the development of basic fabrication processes of the embedded microchannel and nozzle hole on a dual Si AFM tip without cantilever fabrication.

\subsection{Theoretical estimation of liquid flow in microchannel}

Because delivering a small volume of liquid is insufficient with only capillary force in a microchannel, we will apply external pressure to flow a liquid. In a previous report on a hollow AFM cantilever with a rectangular microchannel $\left(2.2 \times 3.7 \mu \mathrm{m}^{2}\right.$ cross section, $155 \mu \mathrm{m}$ long $)$, a flow rate of $50 \mathrm{fL} / \mathrm{s}$ was obtained by applying a $150 \mathrm{kPa}$ pressure to a liquid reservoir. ${ }^{(8)}$ The experimentally applied pressure was much higher than the value (on $10^{2} \mathrm{~Pa}$ order) estimated from the simple fluidic calculation of a rectangular cross-sectional microchannel.

In the case of a microchannel with a circular cross section, the pressure difference between the inlet and the outlet is simply estimated using the Hagen-Poiseuille equation as ${ }^{(9)}$

$$
\Delta P=\frac{8 \eta L}{\pi r^{4}} \times Q
$$

where $\Delta P$ is the pressure difference, $\eta$ is the dynamic viscosity, $L$ is the microchannel length, $r$ is the radius of the microchannel, and $Q$ is the flow rate.

In our microchannel, we need a flow rate of $0.5 \mathrm{fL} / \mathrm{s}$, which corresponds to delivering a droplet of $1 \mu \mathrm{m}$ diameter per second. Our microchannel (2 $\mathrm{mm}$ long) needs a pressure $\Delta P$ of 8 Pa to obtain a $0.5 \mathrm{fL} / \mathrm{s}$ flow rate when the microchannel diameter is $1.5 \mu \mathrm{m}$. Comparing with the reported value $(150 \mathrm{kPa})$, we infer that a sufficient flow rate would be obtained by applying pressure at least on the order of $100 \mathrm{kPa}$, according to the relation that the pressure $\Delta P$ is proportional to $L$ and $1 / r^{4}$.

\subsection{Fabrication of nozzle and microchannel}

Figure 2 shows the schematic structure of the narrow-gapped dual tip. The liquid-delivering cantilever has a nozzle hole at the Si needle tip for liquid delivery. The liquid sample is delivered from a reservoir by external pressure through an embedded channel and dispensed out through a nozzle hole.

The fabrication process of the microchannel and nozzle in a silicon cantilever is shown in Fig. 3. A (100)-oriented single-crystalline bulk $\mathrm{Si}$ wafer was used instead of a silicon-oninsulator (SOI) wafer, which is suitable for accurate cantilever fabrication. Although an actual dual AFM cantilever should be fabricated with an SOI wafer to accurately fabricate a thin $\mathrm{Si}$ cantilever, bulk $\mathrm{Si}$ is used in this study to demonstrate the basic process of dual tip fabrication. First, a (100)-oriented single-crystalline $\mathrm{Si}$ device was thermally oxidized to form a $\mathrm{SiO}_{2}$ film (500 nm thick). Then, the $\mathrm{SiO}_{2}$ film was etched by reactive ion etching (RIE) with an electron 


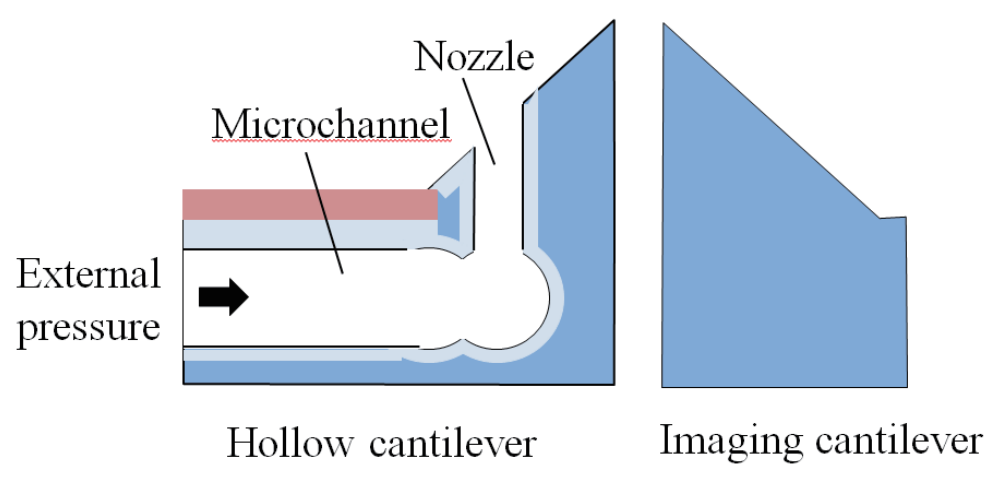

Fig. 2. (Color online) Schematic structure of the narrow-gapped dual tip with liquid delivery function.

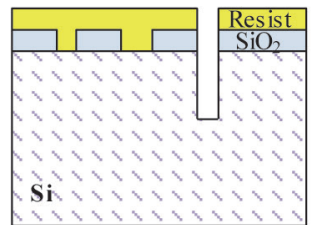

(a) $\mathrm{SiO}_{2}$ patterning

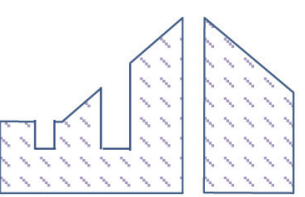

(e) SOG removal

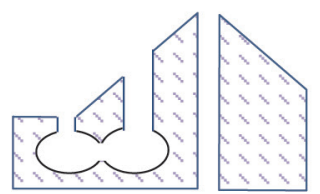

(i) $\mathrm{SiO}_{2}$ film removal

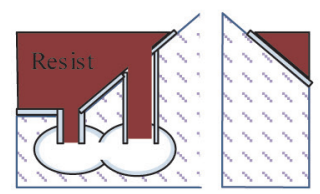

(m) Resist patterning and $\mathrm{SiO}_{2}$ removal for sealing

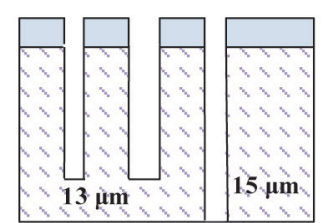

(b) Double mask and DRIE

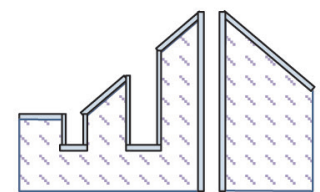

(f) Thermal oxidation

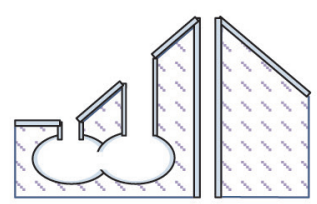

(j) Thermal oxidation

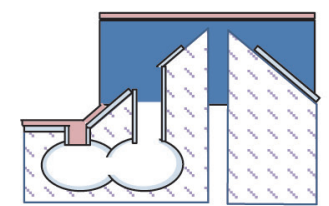

(n) $\mathrm{SiO}_{2}$ sputter deposition

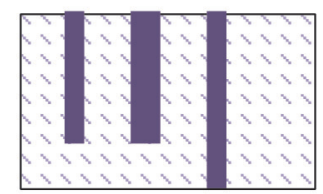

(c) SOG refilling and CMP

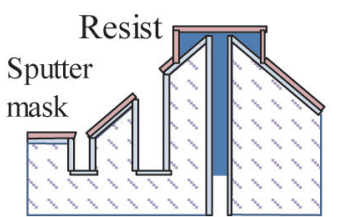

(g) $\mathrm{SiO}_{2}$ sputter deposition for surface protection

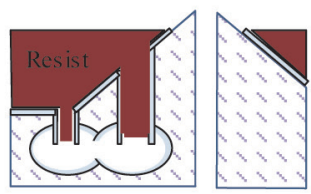

(k) Resist patterning and $\mathrm{SiO}_{2}$ removal

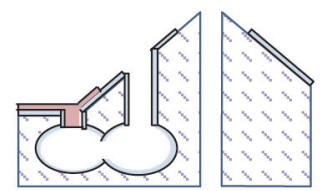

(o) Resist removal and $\mathrm{SiO}_{2}$ lift off

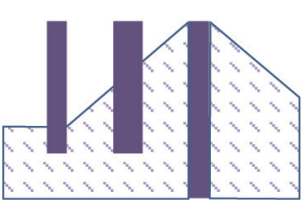

(d) Crystalline anisotropic etching

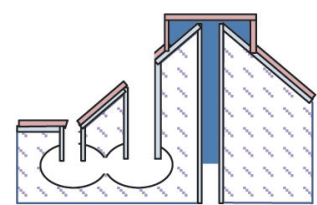

(h) $\mathrm{SiO}_{2}$ etching and $\mathrm{Si}$ isotropic etching

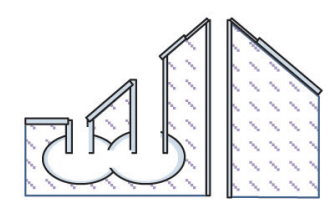

(l) Thermal oxidation at low temperature

Fig. 3. (Color online) Fabrication process of the embedded microchannel and nozzle.

beam (EB) resist mask pattern. After the EB resist removal, Si trenches were formed by RIE using the resist pattern as a mask [Fig. 3(a)]. Si trenches with different depths of 13 and $15 \mu \mathrm{m}$ were formed by DRIE using a double mask, as shown in Fig. 3(b). After the $\mathrm{SiO}_{2}$ film removal, 
the trenches were refilled with spin-on-glass (SOG) to protect the trench sidewalls, followed by chemical mechanical polishing (CMP) to remove the SOG film to expose the Si (100) surface, as shown in Fig. 3(c). Si crystalline anisotropic etching was carried out in tetra-methyl-ammonium hydroxide (TMAH) solution to form inclined (111) crystalline planes to construct narrowgapped twin Si tips [Fig. 3(d)]. The remaining SOG was etched in hydrofluoric acid [Fig. 3(e)]. After the SOG was removed, the trench was covered with a thermally grown $\mathrm{SiO}_{2}$ thin film to protect the trench sidewall [Fig. 3(f)]. After the Si twin tips were protected with a thick negative photoresist pattern (Zeon, ZPN-1150), the $\mathrm{SiO}_{2}$ film was sputtered to protect the Si surface [Fig. 3(g)]. The $\mathrm{SiO}_{2}$ thin film at the trench bottom was selectively removed by $\mathrm{CHF}_{3} \mathrm{RIE}$ and the trench bottom was enlarged by Si plasma isotropic etching to form the microchannels [Fig. 3(h)]. In this isotropic etching process, the nozzle bottom was also enlarged, resulting in the connection to the microchannel under the Si surface. The inner walls of the microchannel and nozzle were thermally oxidized to be covered with a hydrophilic $\mathrm{SiO}_{2}$ thin film [Fig. 3(j)]. The $\mathrm{SiO}_{2}$ film at the probe tip was removed in hydrofluoric acid with a resist mask [Fig. 3(k)]. Thermal oxidation at low temperature $\left(950^{\circ} \mathrm{C}\right)$ was carried out in order to sharpen the Si probe tip [Fig. 3(1)]. The $\mathrm{SiO}_{2}$ thin film at the probe tip was removed [Fig. 3(m)]. After a negative photoresist pattern was formed to protect the $\mathrm{Si}$ needle tips and nozzle hole, a thick $\mathrm{SiO}_{2}$ film was sputtered to completely seal the trench opening above the microchannel, as shown in Fig. 3(n). Finally, a sputtered film on the resist was lifted off by resist removal [Fig. 3(o)].

\subsection{Layout of microchannel and nozzle}

Inclined silicon (111) crystalline planes appear at a concave corner mask pattern when fabricating the probe tips by crystalline anisotropic etching. The appearance of (111) crystalline planes around the probe tip is undesirable, because they also tend to come into contact with a sample surface during the imaging of the sample surface with a Si probe tip. To prevent the appearance of unnecessary (111) planes, layout patterns of the nozzle and microchannels were examined. In this experiment, a Si dual tip was separately located from the nozzle holes. Figure 4(a) shows the etching result when the channel and nozzle were connected to the Si surface. The layout was designed to suppress the (111) plane forming around the nozzle and microchannel connection considering crystal orientation, yet the (111) crystalline plane with a small height appeared at the corner pattern where the nozzle is connected to the microchannel. In the case of the nozzle separated from the microchannel pattern at the Si surface, as shown in Fig. 4(b), no planes were found around the nozzle. The nozzle and microchannel were connected underneath the silicon surface when the bottom parts of the trench and nozzle hole were enlarged by isotropic plasma etching in a subsequent process.

Figure 5 shows the SEM image of a cross section of the fabricated test sample of the enlarged microchannel and nozzle. An array of holes corresponding to the nozzle were located with a gap of $2 \mu \mathrm{m}$, which is sufficiently wide to separate them from their neighboring holes after the $\mathrm{Si}$ isotropic etching, to simplify the cross-sectional observation after Si substrate cleaving. The gap between the hole and the trench was $0.8 \mu \mathrm{m}$. The microchannel of $2.2 \mu \mathrm{m}$ diameter and the nozzle bottom of $2.8 \mu \mathrm{m}$ diameter were fabricated at a depth of $1.5 \mu \mathrm{m}$ below the Si surface. 

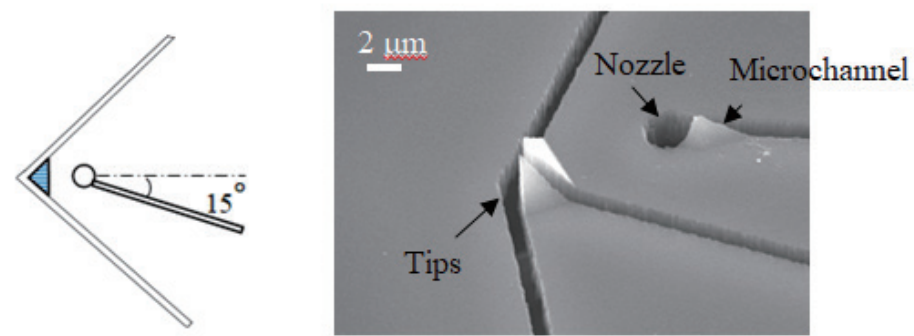

(a)
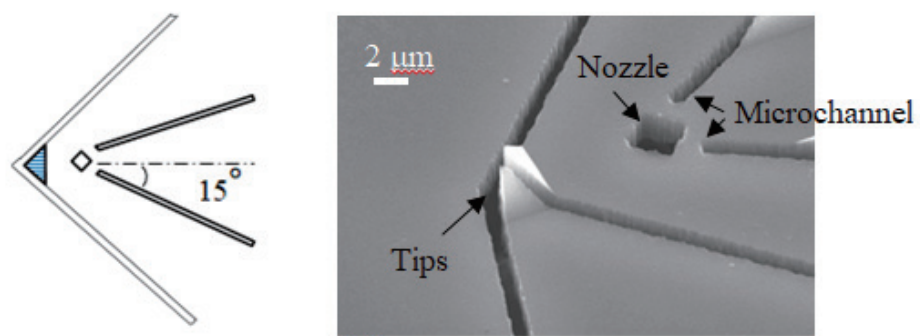

(b)

Fig. 4. (Color online) Layout of nozzle and microchannel (a) connected on the Si surface and (b) separated on the Si surface.

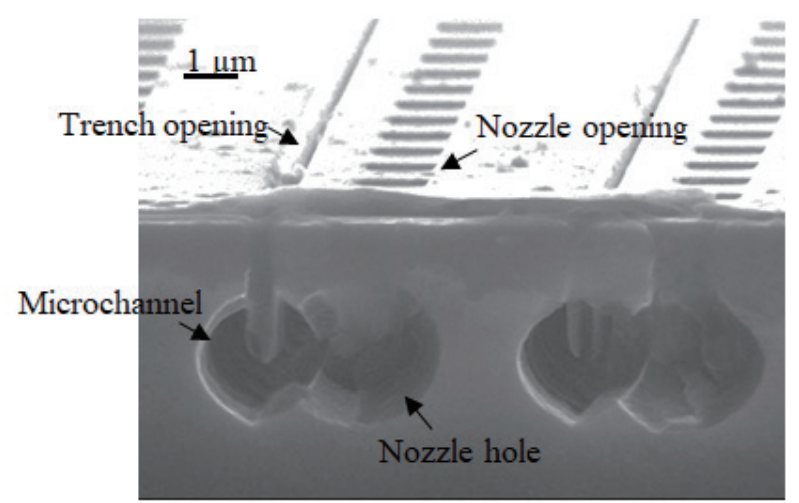

Fig. 5. Cross section of microchannel and nozzle hole connected to each other under the Si surface.

The microchannel and nozzle were separated on the surface. However, they were certainly connected underneath the Si surface.

\subsection{Microchannel sealing}

To complete the hollow cantilever, the trench opening above the microchannel should be sealed. Prior to establishing the fabrication process, preliminary experiments were carried out to seal the opening of the channel using the following three $\mathrm{SiO}_{2}$ film growth methods: 
Si thermal oxidation, chemical vapor deposition, and sputtering deposition. To obtain a large deflection for cantilever switching, it is favorable to minimize the thickness of the sealing film on the cantilever surface and reduce the cantilever stiffness. Also, in the case of a microchannel with a small diameter, a hydrophilic inner wall is preferred for smooth liquid flow. ${ }^{(10)}$ Figure 6(a) shows an example in which the trench (700 nm wide) was completely sealed by the wet thermal oxidation $\left(1100{ }^{\circ} \mathrm{C}\right)$ of a Si film with a thickness of $880 \mathrm{~nm}$. A 660 -nm-thick thermal oxide film was also formed on the inner wall of the microchannel. Although there was the advantage of obtaining a hydrophilic inner wall during the sealing process, the $\mathrm{SiO}_{2}$ film was too thick to maintain the inner channel diameter. The thermal oxidation process also has difficulty in protecting the nozzle hole and Si probe tip from the oxidation. Figure 6(b) shows the microchannel sealed by plasma-enhanced chemical vapor deposition (PE-CVD) using tetraethyl orthosilicate (TEOS) as a source material. The deposition parameters used to deposit TEOS silicon oxide are presented in Table 1. The 660-nm-wide opening was sealed by depositing a 1- $\mu$ m-thick $\mathrm{SiO}_{2}$ film. A thin $\mathrm{SiO}_{2}$ film (about $100 \mathrm{~nm}$ thick) was formed on the inner wall of the microchannel, indicating that the hydrophilic inner wall could be obtained while sealing the channel by TEOS PE-CVD (MPX-CVD, Sumitomo Precision Products Co.). However, it is also difficult to protect the nozzle and Si tip in the CVD process. Figure 6(c) shows the cross section of the microchannel sealed by $\mathrm{SiO}_{2}$ sputter deposition. A 740-nmthick $\mathrm{SiO}_{2}$ film was deposited by reactive sputtering using $\mathrm{Ar} / \mathrm{O}_{2}$ plasma with a $\mathrm{Si}$ target. The 300-nm-wide opening was sealed by depositing a 740-nm-thick $\mathrm{SiO}_{2}$ film. The trench opening was closed by the sputtered $\mathrm{SiO}_{2}$ film before certain deposition into the trench owing to the poor step coverage. In the case of sputtering, the nozzle hole sealing can be avoided by lift-off patterning, but the coverage of the inner wall with a hydrophilic film is poor. The thin thermal oxide film growth mentioned above is usable to obtain the hydrophobic inner wall.

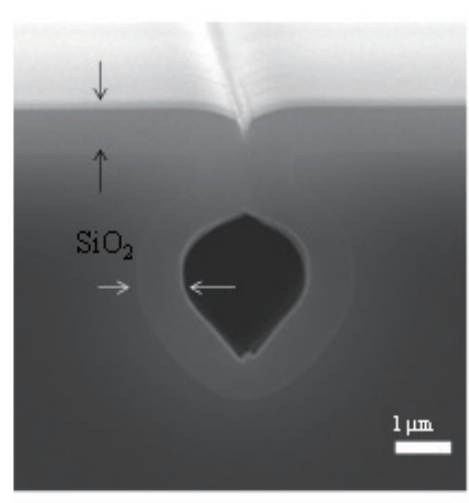

(a)

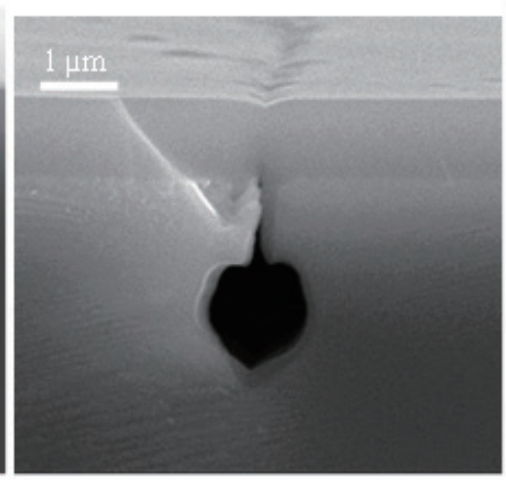

(b)

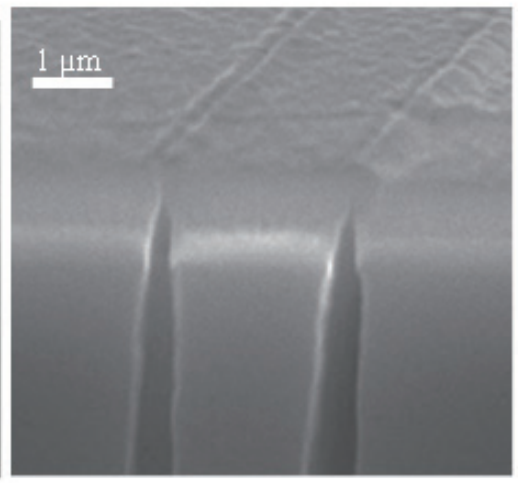

(c)

Fig. 6. Cross sections of microchannels after sealing. (a) Sealed by thermally oxidized $\mathrm{SiO}_{2}$ film growth, (b) sealed by $\mathrm{SiO}_{2}$ film deposition with TEOS PE-CVD, and (c) sealed by sputtered $\mathrm{SiO}_{2}$ film. 
Table 1

Deposition parameters of TEOS PE-CVD.

\begin{tabular}{lc}
\hline Parameters & Conditions \\
\hline RF power $(\mathrm{W})$ & HF (upper electrode): 100 \\
& LF (wafer storage): 20 \\
\hline Pressure $(\mathrm{Pa})$ & 100 \\
\hline \multirow{2}{*}{ Gas flow rate $(\mathrm{sccm})$} & TEOS: 50 \\
& $\mathrm{O}_{2}: 750$ \\
\hline \multirow{2}{*}{ Electrode temperature $\left({ }^{\circ} \mathrm{C}\right)$} & Upper electrode: 250 \\
& Wafer storage: 300 \\
\hline
\end{tabular}

\subsection{Effect of thermal oxidation on Si tip radius}

A sharp probe tip is desirable for high-resolution imaging. It is known that the Si tip can be sharpened by thermal oxidation at a low temperature. ${ }^{(1)}$ However, the thermal oxidation for hydrophilic inner wall formation also affects the sharpness of the probe tip. The change in tip radius through the processes was investigated by high-resolution field emission SEM imaging. The tip radius was measured from a high-magnification SEM image by fitting a circle to the curve. Figure 7(a) shows the initial state of the tip. Figure 7(b) shows the tip after thermal oxidation at $1100{ }^{\circ} \mathrm{C}(250 \mathrm{~nm}$ thick), which was conducted for hydrophilic inner wall formation, and $\mathrm{SiO}_{2}$ film removal in hydrofluoric acid. The tip radius was sharpened to about $30 \mathrm{~nm}$. The tip was further sharpened to below $10 \mathrm{~nm}$ by thermal oxidation at a low temperature ( 950 ${ }^{\circ} \mathrm{C}, 250 \mathrm{~nm}$ thick) for $\mathrm{Si}$ tip sharpening and $\mathrm{SiO}_{2}$ film removal. As a result, it became clear that thermal oxidation at a high temperature for hydrophilic inner wall formation enhances the sharpening of the probe tip.

\subsection{Fabrication of narrow-gapped Si dual tips with embedded microchannel}

The $\mathrm{SiO}_{2}$ film sputter deposition after thermal oxidation $(250 \mathrm{~nm})$ is usable to obtain a hydrophobic and channel sealing, as shown in Fig. 8. Owing to the large nozzle opening (1.2 $\mu \mathrm{m}$ in diameter), the nozzle was not sealed even though the microchannel opening $(500 \mathrm{~nm})$ was completely sealed. After the sealing process, the diameter of the microchannel was 1.5 $\mu \mathrm{m}$. Although the connection path between the nozzle and the microchannel under the surface became slightly narrower when they were $1.2 \mu \mathrm{m}$ apart, which was wider than that indicated in Fig. 5, the connection path with a diameter of about $1 \mu \mathrm{m}$ was successfully obtained. Figure 9 shows the fabricated narrow-gapped Si dual tips with a nozzle, which is connected to the microchannel. The Si tips and the opening of the nozzle hole were preserved during sputter sealing by the resist coverage.

In our future work, we will complete the dual AFM probe with the nozzle and microchannel, and a switching magnetostrictive thin film actuator. Furthermore, we will build an external setup such as a pressure-applying system and a microconnector between an external tube and the tank in the device to demonstrate an actual liquid delivery. 


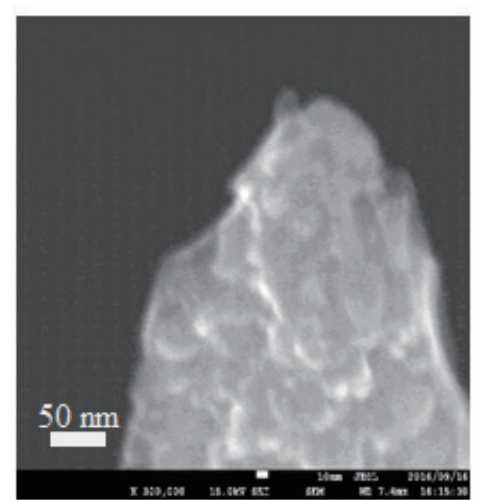

(a)

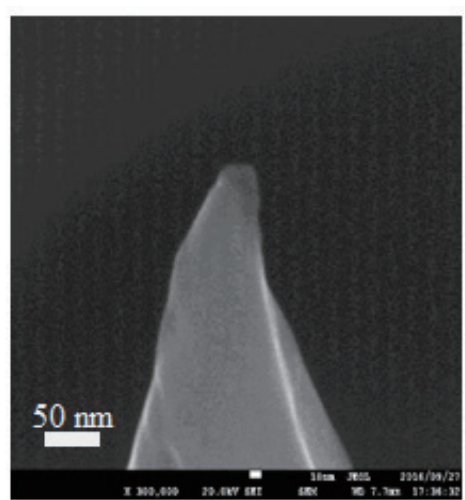

(b)

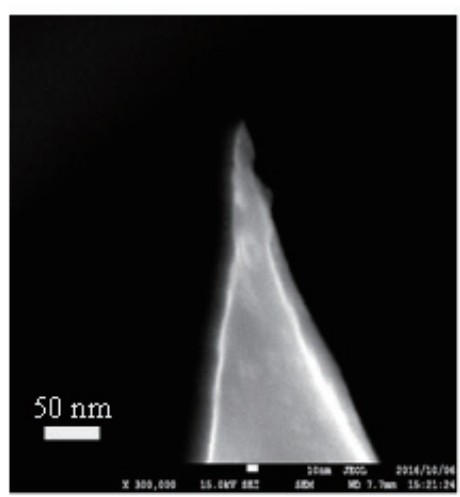

(c)

Fig. 7. Effect of thermal oxidation on tip radius. (a) Before thermal oxidation $(r=80 \mathrm{~nm})$, (b) after thermal oxidation $(r=30 \mathrm{~nm})$ at low temperature, and (c) after thermal oxidation $(r=7 \mathrm{~nm})$.

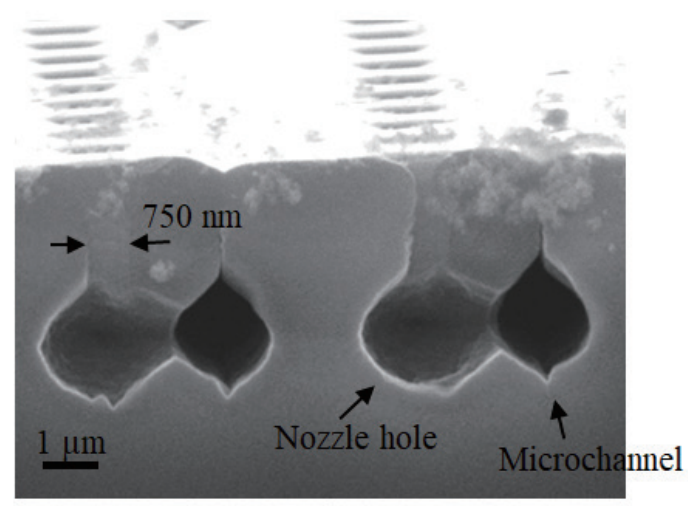

Fig. 8. Cross section of microchannel sealed by sputtered $\mathrm{SiO}_{2}$ film on thermal oxide film.

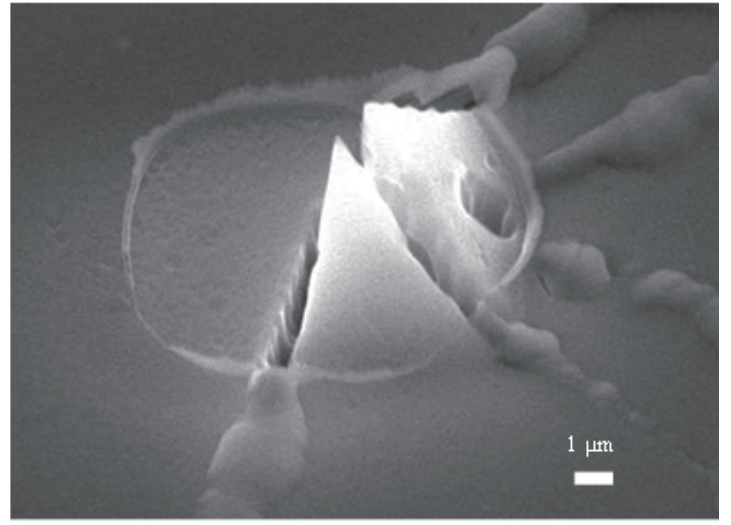

Fig. 9. Narrow-gapped Si dual tips with nozzle and microchannel.

\section{Conclusions}

The basic fabrication processes of dual Si tips with a micronozzle and an embedded microchannel were demonstrated with the aim of realizing a dual hollow AFM cantilever that enables the delivery of a liquid sample to a biomolecule and high-resolution imaging by switching the cantilever.

The fabrication process of a microchannel embedded in a Si cantilever and a nozzle was developed for a narrow-gapped dual Si AFM probe with liquid delivery function. We have designed the layout of the microchannel and nozzle, which were connected underneath the surface to avoid the unnecessary (111) plane appearance at the channel-nozzle connection 
part. The microchannel was completely sealed by the combination of thermal oxidation and sputtering, and the inner wall was coated with a $\mathrm{SiO}_{2}$ film for hydrophilicity. We fabricated the narrow-gapped Si dual tips with a nozzle and a microchannel, and confirmed the connection of the microchannel and nozzle underneath the silicon surface. As a result of our estimation, an applied pressure of $8 \mathrm{~Pa}$ is required when we obtain a flow rate of $0.5 \mathrm{fL} / \mathrm{s}$ through a microchannel of $1.5 \mu \mathrm{m}$ diameter and $2 \mathrm{~mm}$ length. The tip radius can be sharpened by thermal oxidation and further sharpened to a tip radius of $7 \mathrm{~nm}$ by low-temperature thermal oxidation.

In our future work, we will complete the dual AFM cantilever with a liquid-delivering nozzle and a microchannel. We will also build a liquid flow injection setup to demonstrate liquid delivery.

\section{Acknowledgments}

This work was supported by JSPS KAKENHI Grant No. 15H03940 from the Ministry of Education, Culture, Sports, Science, and Technology, Japan. This work was supported by the "Nanotechnology Platform" of the Ministry of Education, Culture, Sports, Science and Technology (MEXT), Japan, at the Center for Integrated Nanotechnology Support, Tohoku University.

\section{References}

1 G. Binnig, C. F. Quate, and Ch. Gerber: Phys. Rev. Lett. 56 (1986) 930.

2 H. H. P. Garza, M. K. Ghatkesar, and U. Staufer: J. Micro-Bio Rob. 8 (2013) 33.

3 T. Shibata, K. Nakamura, S. Horiike, M. Nagai, T. Kawashima, T. Mineta, and E. Makino: Microelectron. Eng. 111 (2013) 325.

4 A. Safi, W. Kang, D. Czapleski, R. Divan, N. Moldovan, and H. D. Espinosa: J. Micromech. Microeng. 23 (2013) 1.

5 K. Kawashima, E. Makino, and T. Mineta: Trans. IEEJ 134 (2014) 74.

6 J. Hong, K. Mishina, and T. Mineta: Trans. IEEJ 138 (2018)

7 Y. Miura, J. Hong, K. Mishina, T. Shibata, and T. Mineta: Proc. 2016 Asia-Pacific Conf. Transducers and Micro-Nano Technology (APCOT, 2016) 233.

8 R. Van Oorschot, H. H. P. Garza, R.J. Derks, U. Staufer, and M. K. Ghatkesar: EPJ T. 2 (2015) 1.

9 Y. Nakayama: Introduction to Fluid Mechanics, R. F. Boucher, Ed. (Yokendo Co. Ltd., Tokyo, 1998) Chap. 6.

10 M. K. Ghatkesar, H. H. P. Garza, F. Heuck, and U. Staufer: Micromachines 2014 (2014) 954.

11 R. B. Marcus, T. S. Ravi, T. Gmitter, K. Chin, D. Lis, W. J. Orvis, D. R. Ciarlo, C. E. Hunt, and J. Trujillo: Appl. Phys. Lett. 56 (1990) 236.

\section{About the Authors}

Jinseo Houg received his M.S. degree in mechanical systems engineering from Yamagata University, Japan, in 2015. He is currently working toward a Ph.D. degree in mechanical systems engineering at Yamagata University, Japan. His research interests are in MEMS, bioengineering, and sensors. (tcr20589@st.yamagata-u.ac.jp)

Yusuke Masuda received his B.S. degree from Yamagata University, Japan, in 2017. He is currently working toward an M.S. degree in mechanical systems engineering at Yamagata University, Japan. (ttf55329@st.yamagata-u.ac.jp) 
Yoshitaka Miura received his B.S. and M.S. degrees from Yamagata University, Japan, in 2014 and 2016, respectively. He joined Tokyo Electron Technology Solutions Ltd. in 2017. (miura-marinesky@i.softbank.jp)

Takayuki Shibata received his B.S. and M.S. degrees in precision engineering from Hokkaido University, Japan, in 1987 and 1989, respectively. He joined Sumitomo Electric Industries from 1989 to 1991 . He was a member of the Faculty of Engineering of Hokkaido University from 1991 to 2001, and the Faculty of Engineering of Ibaraki University from 2001 to 2005. He joined the Department of Mechanical Engineering of Toyohashi University of Technology in 2005. His research interests are in MEMS and nanomachining. (shibata@me.tut.ac.jp)

Takashi Mineta received his B.S. and M.S. degrees in material science from Tokyo Institute of Technology, Japan, in 1984 and 1986, respectively. He received his Ph.D. degree in mechatronics and precision engineering from Tohoku University, Japan, in 2002. He joined the semiconductor group of Toshiba Corporation from 1986 to 1991 and the Yamagata Research Institute of Technology from 1991 to 2004. He was a member of the Faculty of Science and Technology of Hirosaki University from 2004 to 2009. He joined the Faculty of Science and Engineering of Yamagata University in 2010. His research interests are micro/nanofabrication and MEMS-NEMS devices for micro/nanorobotics and medical engineering.

(mineta-t@yz.yamagata-u.ac.jp) 